\title{
Personal Grid
}

\author{
Zhiwei Xu, Lijuan Xiao, and Xingwu Liu \\ Institute of Computing Technology, Chinese Academy of Sciences \\ 100080 Beijing, China
}

\begin{abstract}
A long-term trend in computing platform innovation is the appearance of a new class of platform every 15 years or so, that drastically reduces barriers and expands user base. We have seen this trend in computer's 60-year history several times, with inventions like mainframe, personal computer (PC), Internet, and Web. To explore opportunities brought about by the new net infrastructure, we present a new computing paradigm called Personal Grid (PG). PG allows an individual user to own, control and use a personal server on the net, just as he owns 1a PC today. However, such a virtualized, net-centric server not only enables the user to utilize resources on the net, but also empower the user to contribute to the net and to share and collaborate with other users. We discuss the related emerging workloads and usage modes, the opportunity to lower barriers, the scientific and technical challenges, and research progress made by our Vega Grid Team.
\end{abstract}

\section{Introduction}

The 1960's and 1970's were a golden time in the history of computing system innovation. This wave of innovations helped expand the user population significantly, by reducing the cost barrier, the control barrier, and the knowledge barrier of computing platforms.

System cost was drastically reduced when the market mainstream shifted from mainframes to minicomputers and then microcomputers. Microcomputers coincided with personal computers (PC's), which simultaneously reduced all three barriers. In addition to reducing system cost, each PC is owned and managed (controlled) by a personal user, instead of an enterprise's IT department; and the knowledge required to operate a PC is much simplified compared to a mainframe.

As we enter the 21 st century, the concept of computer has extended to cyber infrastructure, or grids. An opportunity exists to drastically lower the IT cost again, by enabling personally owned and controlled cyberinfrastructure, or personal grids. Cost reduction is possible due to sharing resources in a more efficient architecture. The control barrier can be lowered due to decentralization. The knowledge barrier can be lowered by abstractions and virtualization, and by utilizing man-computer society.

After presenting the personal grid paradigm and a PG architecture, we discuss the emerging workloads and usage modes, the opportunity to lower barriers, the scientific and technical challenges, and research progress made by our Vega Grid Team at Institute of Computing Technology (ICT), Chinese Academy of Sciences. 


\section{Personal Computers, Net Services and Personal Grids}

In addition to the obvious benefit of low system cost, a personal computer has two attractive properties: (1) a PC is a general-purpose computing platform, not a specialpurpose solution such as a calculator; and (2) a PC is personal, meaning that it is controlled by a person (its owner), not an institution. The second property often leads to other properties. For instance, a PC is dedicated, in that a user's application execution on a PC cannot be slowed down by another user.

The net manifests its value to end users usually through various internet services, Web services, and grid services, in a number of overlapping forms:

- Traditional network services, such as email, BBS, and messages.

- Business web, such as salesforce.com.

- Consumer web, such as Amazon and eBay.

- Web 2.0 services, such as Wiki, MySpace, and YouTube.

- Grid services, such as Nanohub.

The current net services have the following characteristics: (1) they provide special-purpose solution services, not a general-purpose platform; (2) they are shared among many users, not for an individual's dedicated use; (3) they are operated and managed by an infrastructure or service provider. In other words, net services today are like the mainframes of the 1960's. They are institutional. The service providers are in control, and each user can only access the services predefined by the providers.

Personal grids combine the nice properties of personal computers and net services. A personal net computing (PNC) environment is shown in Fig. 1, where the PGs are the central piece. Five parties are present. The first layer consists of individual users. Each user uses a client device to access his personal grid.

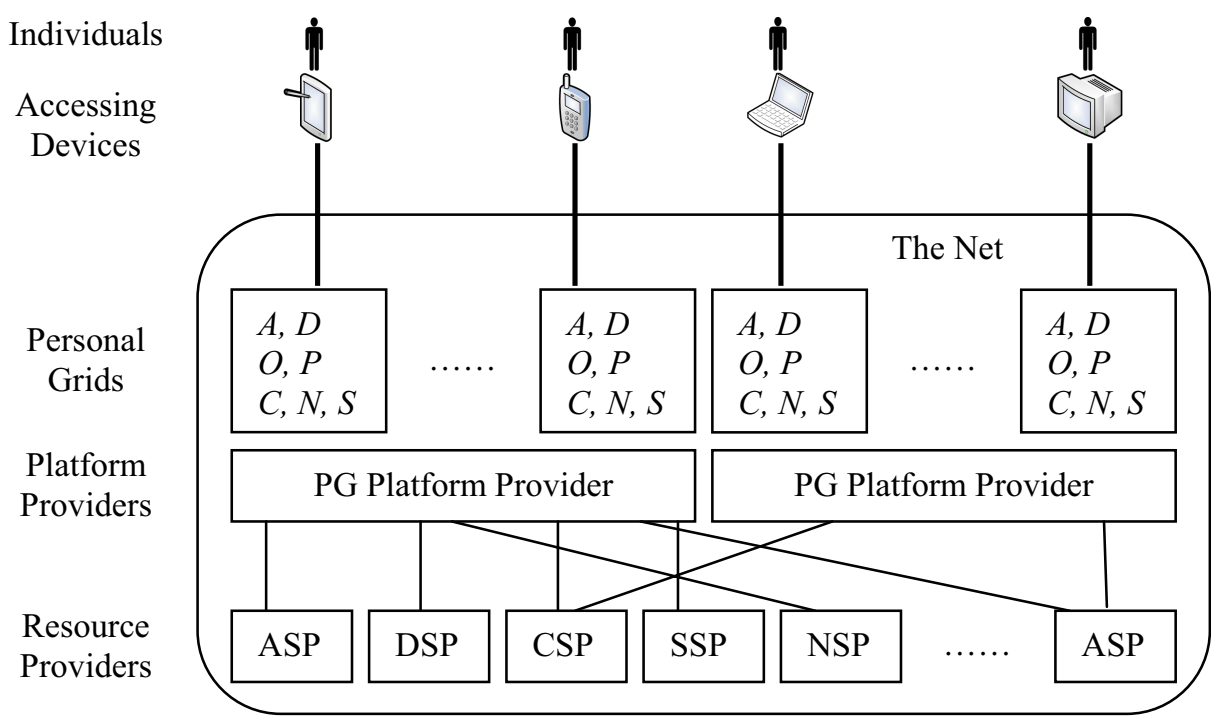

Fig. 1. Personal Net Computing Environment (PNC) Using Personal Grids 
Each PG provides three levels of capabilities to the users. Hardware-level capabilities include computing capability $(C)$, networking capability $(N)$, and storage capability $(S)$. System-level capabilities include operating system operations $(O)$ and policies/contexts $(P)$. Application-level capabilities include application software and services $(A)$ and data $(D)$.

There may be multiple PG platform providers, each providing PGs to the users. The resource providers provide hardware-level and application-level capabilities, usually through some form of net services. The resource types correspond to the capability types visible to the users via a PG. For instance, we have storage service providers (SSP) for the storage capability. Each platform provider can have multiple sources of the same type of resources. Each resource provider can provide for multiple platform providers.

From the user's viewpoint, a personal grid has the following characteristics:

- Platform: A personal grid manifests as a general-purpose computing platform, not a special-purpose solution. This is akin to comparing a personal computer to a calculator.

- Personal: A personal grid is owned and controlled by an individual user, and dedicated to his use.

- Networked: A personal grid is net-centric. Its resources come from the net, and can be shared by other parties on the net.

- Sharing: Users and PGs in a PNC can share the resources from the resources providers, as well as resources from other PGs. The sharing is controlled by the policies/contexts prescribed by each individual user.

- Social: Users and PGs interact and collaborate with one another. In addition to the "raw" resources from the resource providers, the individual users and their PGs can evolve into various clusters and communities, where social assets (social resources) common to a community will be accumulated.

- Decentralized: The PNC is a decentralized computing platform, where each user gets a slice of it (the user's PG). The PGs are independent. The shutdown of any resource provider will not make a personal grid unavailable.

\section{Opportunities and Challenges}

An obvious opportunity is to study and utilize the new workload and usage mode characteristics brought about by the personal grid paradigm. The five layers in Fig. 1 may see quite different workloads and usage modes. Moreover, the following types of workload may become increasingly important:

- Dynamic workloads from applications with scripting, dynamic libraries, XML interpretation, and Java codes.

- Interaction operations from man-machine, man-man, and machine-machine collaborations.

- Metadata operations for individual personal grids, clusters and communities.

- Policy operations for contexts, security, reputation, trust, and privacy.

- Discovery operations like browsing, searching, and tags computing.

- Market operations such as classified sales, recommendation, and bidding. 
The second opportunity is to reduce the large amount of over-provisioning, redundancy, idleness, overhead, and other inefficiencies, thus lowering the per user cost. As each PG is a virtualized server on the net, resource over-provisioning can be avoided by on-demand, good-enough provisioning. There is also much space of improvement for individual requests, and still larger potential for improving multiple requests from many users and PGs. Our preliminary experiments show that fetching 10-byte data from a blog server takes $162 \mathrm{~ms}$ and 52 context switches at server side, where PHP scripts execution alone accounts for $96 \%$ of the total time. To call an empty Web service in Tomcat+Axis needs $15 \mathrm{~ms}$. Calling a grid service with security needs 0.5-2 seconds. Such excessive overheads offer innovation opportunities.

Cost reduction may come from another source: the commoditization of resource providers. Solution-level net services of today, turned into capabilities, will become commodities, each type with multiple vendors. This will encourage competition and innovation. The same happened before for PCs. It is likely to happen with PGs. A loosely coupled architecture and standard interfaces are technical enablers.

The third opportunity lies in decentralization. The PGs and the resource providers are both decentralized. Thus independent innovation and advances are possible. The use and management of these two parties are also independent. The absence of architectural inter-dependency help lower the control barrier. Users and resource providers have more freedom in the control/use/provisioning of their own capabilities, and in how to integrate/collaborate with others. This is unlike many net services today, where users' data are tied to a specific application. Only limited interaction and integration are possible, via techniques such as mashup.

The fourth opportunity is the PG user interface, which is a key to reduce the knowledge barrier. The desktop and the browser metaphors may be augmented with new metaphors. In fact, a number of complementary metaphors may be created to support PGs. For instance, to manage a PG's capabilities, a banking metaphor may be appropriate. Each user (thus each PG) may have a capability portfolio, consisting of several personal accounts of different types of capabilities. A user may save cash capabilities for her current needs, invest excess capabilities, or borrow capabilities.

The opportunities are accompanied with many technical challenges. Consider the efficiency challenge. To lower the cost, the PNC architecture in Fig. 1 must be efficiently implemented and used, so that resources are efficiently utilized, with effective sharing and reuse.

A PG should have the responsiveness of a PC. Normal commands and interactive operations should have short response times (e.g., less than 0.25 seconds). However, a PG may need virtualization and high-level abstractions. User visible commands and operations of a PG thus may contain much more substance. As discussed above, even a small part of such a command will need hundreds of milliseconds to over a second. One reason is that such a "simple" operation needs to traverse a tall stack of layers, from application logic to WSDL, SOAP, XML, HTTP, TCP/IP to operating system calls. How to reduce layering overhead is a major technical challenge.

We also want a PG to be dedicated for a user. The performance should not be visibly affected when many users are using the underlying infrastructure and its resources. That is, we want PGs that are virtually dedicated to their individual owners, even though they may physically share resources. Simultaneously achieving such sharing and isolation for personal grids brings new challenges, especially considering 
that PNC is a decentralized environment. There is no centralized, omnipotent operating system kernel to help.

Users may access their personal grids via all kinds of devices with variant quality of network connection. How to tolerate transient disconnection and to enable offline operation are research issues. Data hoarding and proper client side running of PG applications are needed. Mining such workload characteristics could significantly improve user experience.

Another major challenge is to attract users and resource providers to join and stick with the personal grids (or the PNC). What are the proper incentives for them? Can these incentives be effectively implemented? Can the users and resource providers conveniently use such incentives? This is not only a business model question. There are technical and scientific issues involved.

From a scientific viewpoint, a very interesting research area has to do with emergent properties. PGs and PNC form a decentralized computing environment. Users and resource providers are highly autonomous. They make local decisions and have access to mostly local or community information. What global, emergent properties can arise? What are the necessary and sufficient conditions for "nice" emergent properties? Are there effective, constructive techniques that can implement such conditions?

\section{Technology}

At ICT, the following issues are being studied to realize personal grids:

Usage Classification. We have organized the PG usage modes into two classes of six types. The first class is for personal use, including three types of usage modes for consumption, management and production of resources. The second class is for social use, which includes three types of usage modes for sharing, context and contribution of resources, policies and contents.

System abstractions. We are developing a very small set of abstractions to support the common requirements of personal grids. For instance, each PG is implemented as a container of persistent handles, connecting to virtualized resources.

Virtualization. While each user sees a PG owned and controlled by herself, it is a virtualized set of capabilities, realized by resource providers. Techniques are being developed for efficient virtualization, such that direct (non-layered) execution is possible in most cases.

New forms of locality. We are exploring new locality phenomena, such as request locality (requests from independent users may hit the same resources) and social locality (e.g., locality in contexts, clusters, policies), to improve the efficiency of PGs.

Emergent properties. We are studying various potential emergent properties and the ways to achieve them, such as fair market and balanced utilization.

Acknowledgements. This work is supported in part by the National Natural Science Foundation of China (Grant No. 90412010, 60603004), the China National 973 Program (Grant No. 2005CB321807), and the Chinese Academy of Sciences Distinguished Scholars Fund (Grant No. 20014010). 